\title{
Note Correcting a Remark in a Paper of Karl Bosch
}

\section{Rosenblatt}

Department of Mathematics, University of California, Box 109, San Diego, La Jolla, Cal. 92093, USA

In a paper "Notwendige und hinreichende Bedingungen dafür, daß eine Funktion einer homogenen Markoffschen Kette Markoffsch ist" appearing in vol. 31, 199-202 (1975) of this journal, Karl Bosch states that Theorem 2 of a paper of C.J. Burke and myself "A Markovian function of a Markov chain" (p.1113 Ann. Math. Statist. 29 (1958)) is false. A careful reading of the proof of Theorem 2 shows that Theorem 2 is correct as the proof involves a simple verification of the Markov property. Bosch in his argument looks at an example of a Markov chain we considered with transition matrix

$$
\left(\begin{array}{ccccc}
\frac{1}{3} & \frac{1}{6} & \frac{1}{3} & \frac{1}{6} & 0 \\
\frac{1}{6} & \frac{1}{3} & \frac{1}{6} & \frac{1}{6} & \frac{1}{6} \\
\frac{1}{4} & 0 & \frac{1}{4} & \frac{1}{6} & \frac{1}{3} \\
\frac{1}{4} & \frac{1}{4} & \frac{1}{4} & 0 & \frac{1}{4} \\
0 & \frac{1}{4} & 0 & \frac{1}{2} & \frac{1}{4}
\end{array}\right)
$$

and any initial distribution $w_{1}, w_{2}, w_{3}, w_{4}, w_{5}$. The states $1,2,3$ are collapsed into a single state $S$. The apparent error in Bosch's argument is the assumption that the collapsed process, if it is a Markov chain, will be homogeneous. In the example the collapsed Markov chain which is Markovian for any initial distribution, will be homogeneous if and only if $w_{1}=w_{3}$. 